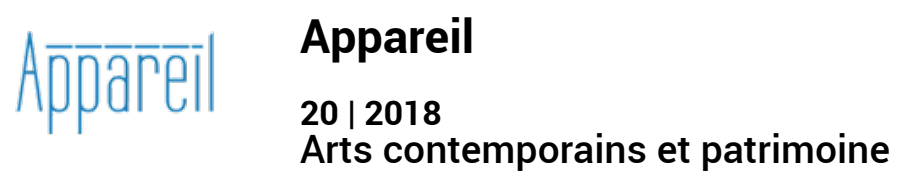

\title{
Coutures et cortèges
}

À propos de l'exposition de Sylvie Villaume, «Les patients » (15/IX- fin octobre 2015), présentée dans la nef de l'hôtel-Dieu de Tonnerre

Daniel Payot

\section{OpenEdition}

Journals

Édition électronique

URL : http://journals.openedition.org/appareil/2423

DOI : 10.4000/appareil.2423

ISSN : 2101-0714

Éditeur

MSH Paris Nord

Référence électronique

Daniel Payot, "Coutures et cortèges », Appareil [En ligne], 20 | 2018, mis en ligne le 26 octobre 2018, consulté le 30 juillet 2020. URL : http://journals.openedition.org/appareil/2423 ; DOI : https://doi.org/ 10.4000/appareil.2423

Ce document a été généré automatiquement le 30 juillet 2020.

\section{(c) (i) () $\Theta$}

Appareil est mis à disposition selon les termes de la Licence Creative Commons Attribution - Pas d'Utilisation Commerciale - Pas de Modification 4.0 International. 


\section{Coutures et cortèges}

À propos de l'exposition de Sylvie Villaume, «Les patients » (15/IX- fin octobre 2015), présentée dans la nef de l'hôtel-Dieu de Tonnerre

\section{Daniel Payot}

1 La poétique de Sylvie Villaume ${ }^{1}$ semble constitutionnellement préservée de toute emphase. Elle suit sa route comme si, étrangère à toute séduction de l'originel et de l'ultime, elle s'était depuis toujours libérée des contraintes liées à des désirs d'accomplissement. Chez elle, pas de fantasme de l'œuvre unique, imposante, définitive. Pas d'intention de subjuguer, conquérir, dominer, soumettre, enchaîner. Sa route évite les monuments sublimes, esquive les promesses de miracle ou autres événement prodigieux, s'écarte des parages où s'annoncent apparitions sacrées et couronnements absolus.

2 Elle ne dédaigne pas les sites magnifiques, mais c'est pour leur rendre hommage et non pour profiter de leur prestige et les enrôler comme décors ou écrins. Elle se demande ce qu'ils sont, eux, en eux-mêmes, quelles traces ils portent, quelles mémoires ils recèlent, quelles souffrances et quelles espérances s'y sont dites, de quels oublis aussi ils sont devenus le théâtre ou la salle des machines. Elle se confronte alors, jamais pleinement assurée de ses propres suppositions, aux spectres qui les traversent, qui évoquent leurs morts et parfois font revenir leurs vivants délaissés, qui rejouent les drames et les bonnes fortunes dont ces lieux furent et sont parfois encore les abris et les témoins.

3 Font alors leur entrée, sans crier gare, sans tambour ni trompette, loin de tout apparat, soustraits à tout signe extérieur de richesse, de pouvoir, d'importance protocolaire, démunis de toute officialité comme de tout orgueil olympien, des populations non uniformes, des cortèges peu assortis, des hordes tangiblement disparates. Leur défilé hétérogène exprime au premier abord une pauvreté légère, ludique, fière et rieuse. On devine le plaisir malicieux que prend chaque élément singulier quand il constate que son apparence est toute différente de celle de ses compagnons d'aventure et que leurs ressemblances fortuites ne les rendent jamais identiques. Chacun est comme réparé, récemment raccommodé, constitué de pièces éparses précairement assemblées, et cet assemblage, loin d'être signe de misère, devient pour chaque individu du groupe la 
preuve de sa reconnaissance et l'indice de sa liberté sans bornes au cœur de l'ensemble accueillant auquel il contribue.

Ce ne sont pas de lourds troupeaux terriens, mais des vols d'oiseaux dans l'éther. Chacun fend l'air sans revendiquer d'y marquer son territoire, et ensemble ils dessinent des figures complexes qu'ils ne ressentent pas le besoin de fixer ni de signer. Leurs ascensions n'ont rien de religieux ni de métaphysique, leurs chutes et leurs piqués rien de catastrophique, ce sont seulement des façons de jouer avec les trois dimensions. Ce qui n'exclut pas mémoire, évocation et sens. Ces élans, ces tourbillons, ces parades se déroulent parfois dans des lieux qui furent de douleur et d'espoir, d'injustice et de solidarité, de répression et de lutte. Leur légèreté n'est jamais indifférence.

Dans le magnifique vaisseau de l'Hôtel-Dieu de Tonnerre, ce sont peut-être des générations d'âmes de patients et de sœurs soignantes depuis 1295, peut-être aussi celles des défunts qui y furent inhumés aux XVII et XVIII siècles qui reviennent, se redressent, s'assemblent en un cortège simultanément grinçant et rieur. Leur spectrale insistance est sans doute bien éloignée de toute véracité figurative - mais qui pourrait prétendre connaître précisément le visage des âmes? Elles adoptent ici une posture principalement verticale, comme si une nostalgie d'être debout les mettait en marche. Elles sont relevées, comme le sont les inscriptions gravées sur les tombes qui, rapportées par frottage puis transposées sur des draps, rejoignent à leur tour l'ondoyant pèlerinage. Aucune ne retrouve le contact du sol, ne le foule, ne le marque ni ne s'appuie sur lui pour rebondir, comme si la progression et le mouvement s'étaient délibérément émancipés de toute pesanteur et de toute adhérence. Leur suspension transforme en vol d'oiseau la succession de leurs pas, elle ménage aussi sous elles l'écart nécessaire, l'intervalle, la distance indéchiffrable sans lesquels leur forme serait la représentation suffisante de quelque chose plutôt que la suggestion imparfaite et inépuisable d'un souvenir ailé.

6 La poétique de Sylvie Villaume substitue l'intrigue à l'emphase. À la magnificence de l'unique elle oppose la diversité illimitée d'éléments hétérogènes qu'elle n'abandonne pas à leur solitude chagrine, mais qu'elle tresse, entremêle, tisse en des assemblages toujours singuliers et toujours incertains, combinaisons improbables, mosaïques dépareillées, échafaudages vacillants. C'est une poétique de l'invention, au double sens du terme : elle se nourrit de ce qu'elle trouve, ramasse, recueille et relève, elle héberge et honore un déjà-là existant tenu en lui-même pour un réservoir infini de sens et de formes ; et elle associe, crée des liens, des voisinages inédits, concordances, contiguïtés, mitoyennetés, Witz et rapprochements incongrus qui font sens aussi par eux-mêmes. Cela ne tournerait que de plaisantes anecdotes si ces associations n'étaient qu'ingéniosités arbitraires: mais il se trouve que leurs significations immanentes consonent avec celles de l'existant recueilli, et c'est là toute la dynamique, tout le suspens de l'intrigue, qui n'est pas seulement collage mais récit, pas seulement trouvaille statique mais déploiement temporel. La poétique de Sylvie Villaume est une poétique de la couture (même en volume) et du cortège : les choses singulières qu'elle bricole, monte ou ravaude sont tout de suite destinées à former pelotes et cohortes, les entrelacs matériels, raccords, faufils, surfils et surjets qui font être chaque objet dans l'espace ont pour répondant immédiat les entremêlements que ces objets composent ensemble, et ces tissages de tissus, ces entretressements de tresses ne tiennent pas en place, se mettent aussitôt en marche, circulent, se font passages et déambulations. 
7 Ces écritures dans l'espace et dans le temps sont prosaïques et poétiques, discursives et narratives, elles ne sont jamais démonstratives ni normatives. Nous voyons défiler des théories de formes rafistolées et des constellations d'objets impertinents, et aucune jamais n'est l'illustration, l'exemple ou l'actualisation de quelque modèle idéal ou de quelque structure sous-jacente. Comme l'écrivain défini par Gilles Deleuze, Sylvie Villaume "invente des agencements à partir des agencements qui l'ont inventé[e] ${ }^{2}$ ", elle "fait passer une multiplicité dans une autre", elle fait "conspirer tous les éléments d'un ensemble non homogène » qu'elle laisse «fonctionner ensemble » sans que leurs réunions aient à répondre de quelconques « conditions d'homogénéité ».

8 Ce ne sont pas des identités et des essences qu'elle relie, mais, disait étrangement Deleuze à propos de l'écrivain, « des noms [...] de peuples et de tribus, de faunes et de flores, d'opérations militaires et de typhons, de collectifs, de sociétés anonymes et de bureaux de production", ou encore "des collections de sensations intensives», des " paquets de sensations à vif ", collections et combinaisons qui « filent sur des lignes de chance, ou de malchance, là où se font leurs rencontres, au besoin leurs mauvaises rencontres ».

De «l'agencement» ainsi compris, Gilles Deleuze disait qu'il était «l'unité réelle minima » de la production d'énoncés et qu'il était « toujours collectif », qu'il mettait en jeu, « en nous et hors de nous, des populations, des multiplicités, des territoires, des devenirs, des affects, des événements ". Peut-être n'est-il pas tout à fait arbitraire d'emprunter le mot au philosophe et de conclure alors : la poétique de Sylvie Villaume récuse emphase et unicité parce qu'elle est une poétique de l'agencement. Ou plutôt, car on ne voit pas comment on pourrait ne pas mettre au pluriel ce terme-là aussi et comment on pourrait l'abandonner à un orgueilleux isolement: une poétique tissée d'intrigues et d'agencements.

\section{NOTES}

1. 1 Voir « Les patients » de Sylvie Villaume - performArts.

2. Les propos cités dans les derniers paragraphes sont extraits de Gilles Deleuze et Claire Parnet, Dialogues, Champs Flammarion, 1996, p. 51 et p. 65.

\section{AUTEUR}

\section{DANIEL PAYOT}

Daniel Payot est professeur à l'Université de Strasbourg et membre de l'unité de recherche ACCRA - Approches contemporaines de la création et de la réflexion artistiques. 